\title{
Profiling the miRNA-mRNA-IncRNA interaction network in MSC osteoblast differentiation induced by (+)-cholesten-3-one
}

\author{
Qiuke Hou ${ }^{1,2+}$, Yongquan Huang ${ }^{3 \dagger}$, Yamei Liư ${ }^{4}$, Yiwen Luo ${ }^{5}$, Bin Wang ${ }^{5}$, Rudong Deng ${ }^{1}$, Saixia Zhang ${ }^{1}$,
} Fengbin Liu $^{2}$ and Dongfeng Chen ${ }^{1 *}$

\begin{abstract}
Background: Our previous study showed that (+)-cholesten-3-one $(\mathrm{CN})$ has the potential to induce the osteoblastic differentiation of mesenchymal stem cells (MSCs). However, the roles of CN in targeting miRNA-mRNA-IncRNA interactions to regulate osteoblast differentiation remain poorly understood.

Results: A total of 77 miRNAs (36 upregulated and 41 downregulated) and 295 IncRNAs (281 upregulated and 14 downregulated) were significantly differentially expressed during CN-induced MSC osteogenic differentiation. Bioinformatic analysis identified that several pathways may play vital roles in MSC osteogenic differentiation, such as the vitamin D receptor signalling, TNF signalling, PI3K-Akt signalling, calcium signalling, and mineral absorption pathways. Further bioinformatic analysis revealed 16 core genes, including 6 mRNAs (Vdr, Mgp, Fabp3, Fst, Cd38, and Col1a1), 5 miRNAs (miR-483, miR-298, miR-361, miR-92b and miR-155) and 5 IncRNAs (NR_046246.1, NR_046239. 1, XR_086062.1, XR_145872.1 and XR_146737.1), that may play important roles in regulating the CN-induced osteogenic differentiation of MSCs. Verified by the luciferase reporter, AR-S, QRT-PCR and western blot assays, we identified one miRNA (miR-298) that may enhance the osteogenic differentiation potential of MSCs via the vitamin D receptor signalling pathway.

Conclusions: This study revealed the global expression profile of miRNAs and IncRNAs involved in the Chinese medicine active ingredient CN-induced osteoblast differentiation of MSCs for the first time and provided a foundation for future investigations of miRNA-mRNA-IncRNA interaction networks to completely illuminate the regulatory role of CN in MSC osteoblast differentiation.
\end{abstract}

Keywords: (+)-cholesten-3-one, Osteoblastic differentiation, Mesenchymal stem cells, miRNA-mRNA-IncRNA

\section{Background}

Mesenchymal stem cells (MSCs) are the most promising bone regeneration and repair cells due to their osteogenic potential [1]. Though it has great potential for clinical applications [2], the osteogenic differentiation of MSCs is precisely regulated and coordinated by mechanical and molecular signals from the extracellular environment and involves complex pathways at the transcriptional and post-transcriptional levels $[3,4]$ that

\footnotetext{
*Correspondence: cdf27212@21cn.com

${ }^{\dagger}$ Qiuke Hou and Yongquan Huang contributed equally to this work.

'Department of Anatomy, The Research Centre of Integrative Medicine,

Guangzhou University of Chinese Medicine, Guangzhou 510000, Guangdong,

People's Republic of China

Full list of author information is available at the end of the article
}

remain largely unexplored. Steroids, especially (+)-cholesten-3-one $(\mathrm{CN})$, have therapeutic potential to enhance the osteoblast differentiation of MSCs and increase the expression of osteogenesis-specific factors, as reported in our previous study $[5,6]$. However, previous studies were focused on the transcriptional, post-transcriptional and epigenetic levels, and the exact molecular mechanisms involved in osteogenesis and pre-transcriptional levels require further study.

MicroRNAs (miRNAs) are a class of small-molecule (17-25 nucleotides), single-chain RNAs that do not encode any proteins and have been identified as regulators of biological processes $[7,8]$. MiRNAs regulate gene expression mainly by binding to the 3 '-untranslated regions 
(3'-UTRs) of target mRNAs, resulting in their degradation or inhibition of their translation $[9,10]$, and miRNAs can also regulate gene expression by binding to $5^{\prime}$-UTR [11, 12], even in coding regions. More recently, advances in high-throughput technologies and computational methods have enabled unprecedented analysis of long-chain noncoding RNAs (lncRNAs) (another class of noncoding RNAs) [13, 14]. Increasing evidence [15-17] has shown that lncRNAs regulate gene expression by interacting with DNA, RNA or proteins, but identifying the functions of individual lncRNAs remains challenging [18-20]. Previous research has focused on exploring how noncoding RNAs regulate mRNA expression; however, some scholars believe that miRNAs, lncRNAs and mRNA can form a well-regulated interaction network $[21,22]$. Previous studies $[21,22]$ have reported that miRNA-mRNA-lncRNA interaction networks play important roles in biological processes. However, none of these interaction networks have been shown to regulate the $\mathrm{CN}$-induced osteoblast differentiation of MSCs. Therefore, further research is required to investigate the miRNA-mRNA-lncRNA interaction network in the $\mathrm{CN}$-induced osteoblast differentiation of MSCs.

Herein, microarray analysis was used to explore the differential expression of miRNAs and lncRNAs during $\mathrm{CN}$-induced MSC osteoblast differentiation. Subsequently, bioinformatics analyses and in vitro experiments were conducted to explore the miRNA-mRNA-lncRNA interaction network during $\mathrm{CN}$-induced MSC osteoblast differentiation. Our findings lay the basis for future investigations of the miRNA-mRNA-lncRNA interaction network in MSC osteogenesis regulated by $\mathrm{CN}$.
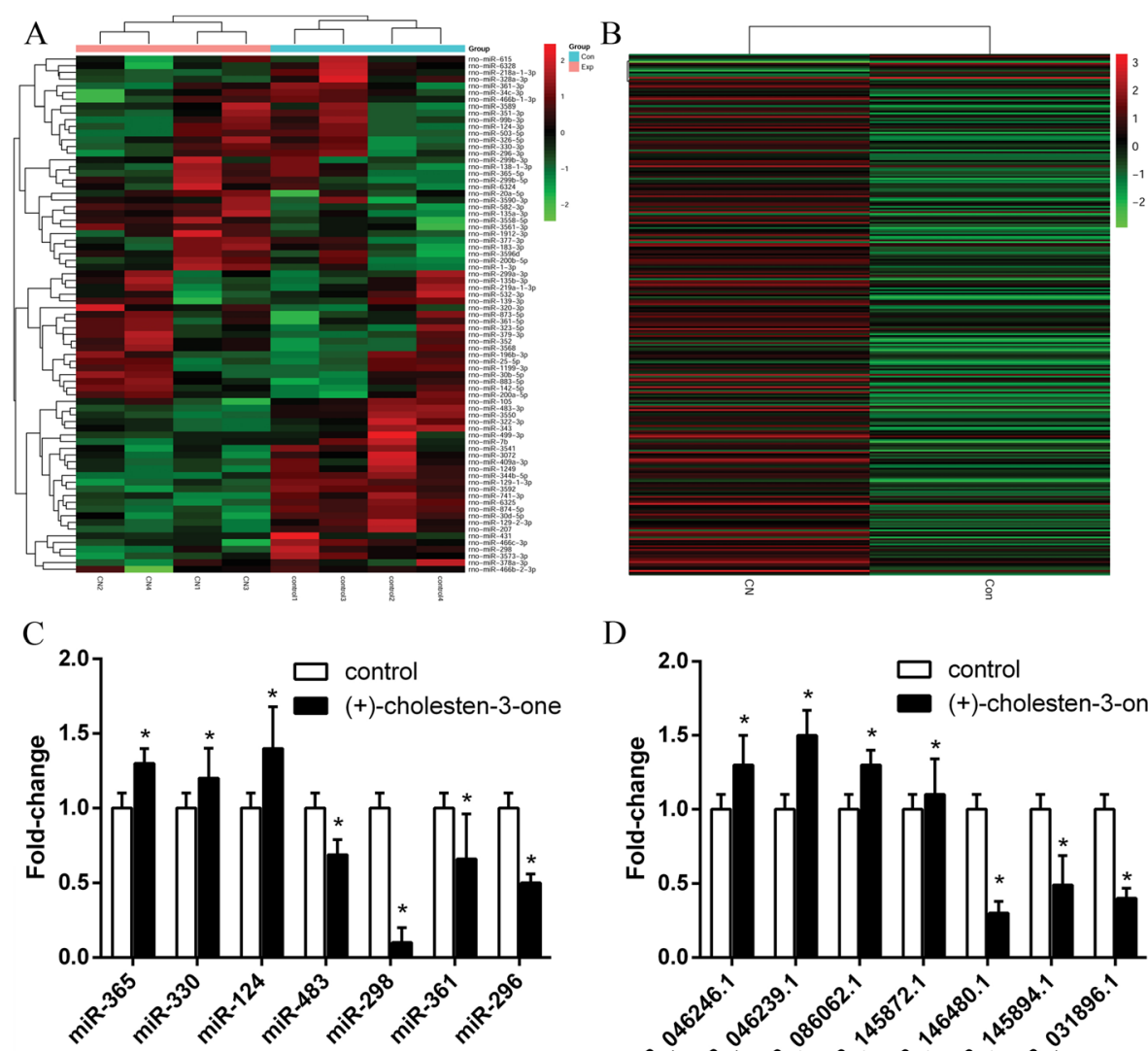

D

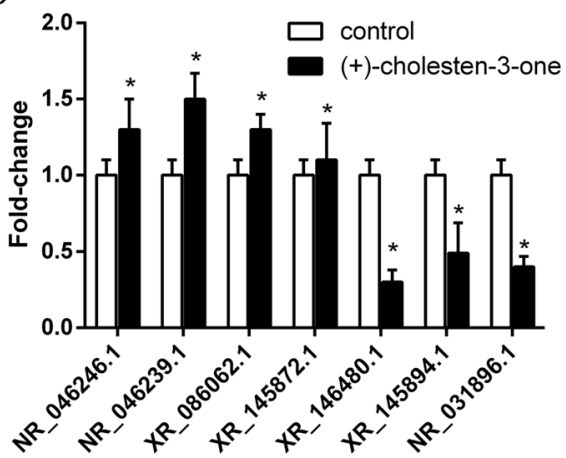

Fig. 1 Heat map of miRNA and IncRNA differential expression during the CN-induced osteoblast differentiation of MSCs. a In total, 77 miRNAs were differentially expressed, including 36 that were upregulated and 41 that were downregulated. $\mathbf{b}$ In total, 295 IncRNAs were differentially expressed, including 281 that were upregulated and 14 that were downregulated. c qRT-PCR verification of the miRNA microarray results. Compared with the control group, the expression levels of miR-365, miR-330 and miR-124 were higher in CN-induced MSCs, and those of miR-483, miR-298, miR-361 and miR-296 were lower in CN-induced MSCs, which was consistent with the miRNA microarray results. $\mathbf{d}$ qRT-PCR verification of the IncRNA microarray results. Compared with the control group, the expression levels of NR_046246.1, NR_046239.1, XR_086062.1, and XR_145872.1 were higher in CN-induced MSCs, and those of NR_031896.1, XR_146480.1 and XR_145894.1 were lower in CN-induced MSCs, which was consistent with the IncRNA microarray results. $P<0.05$, compared to the control group. The color scale shown on the top illustrates the relative expression level of the genes between two group: red denotes high expression levels, whereas green denotes low expression levels 


\section{Results}

Differentially expressed miRNA and IncRNA profiles during $\mathrm{CN}$-induced MSC osteoblastic differentiation

A total of 77 differentially expressed miRNAs were identified during $\mathrm{CN}$-induced osteoblast differentiation compared to uninduced MSCs $(P<0.05)$; among them, 36 were upregulated and 41 were downregulated. In addition, a total of 295 lncRNAs were differentially expressed after the $\mathrm{CN}$-induced osteoblast differentiation of MSCs $(P<0.05)$, of which 281 were upregulated and 14 were downregulated (Fig. 1a \& b; Additional file 1).

Seven miRNAs (miR-483, miR-298, miR-361, miR-296, miR-365, miR-330 and miR-124) and 7 lncRNAs (NR_046246.1, NR_046239.1, NR_031896.1, XR_086062.1, XR_146480.1, XR_145872.1 and XR_145894.1) were randomly selected to verify the reliability of the microarray data via qRT-PCR, which showed results similar to those of the microarray profiles (Fig. 1c \& d).
Candidate mRNA prediction and coding-noncoding gene co-expression (CNC) network construction during $\mathrm{CN}$-induced MSC osteoblast differentiation

Because the differential expression of mRNAs during $\mathrm{CN}$-induced MSC osteoblast differentiation was not detectable in this study, mRNA predictions were made using miRanda (http://miranda.org.uk/) based on the miRNA and lncRNA microarray. A total of 443 possible mRNAs were predicted to targeted by differentially expressed miRNA and lncRNA during $\mathrm{CN}$-induced osteoblast differentiation compared to uninduced MSCs $(P<0.05)$; of these, 223 were predicted to be upregulated, and 220 were predicted to be downregulated $(P<0.05)$ (Additional file 2$)$. To confirm the reliability of the predicted target genes, 7 mRNAs (Vdr, Mgp, Fst, Cd38, Nln, Rfc3 and Car9) were selected for validation with qRT-PCR, which showed results similar to the predicted target genes (Fig. 2a).
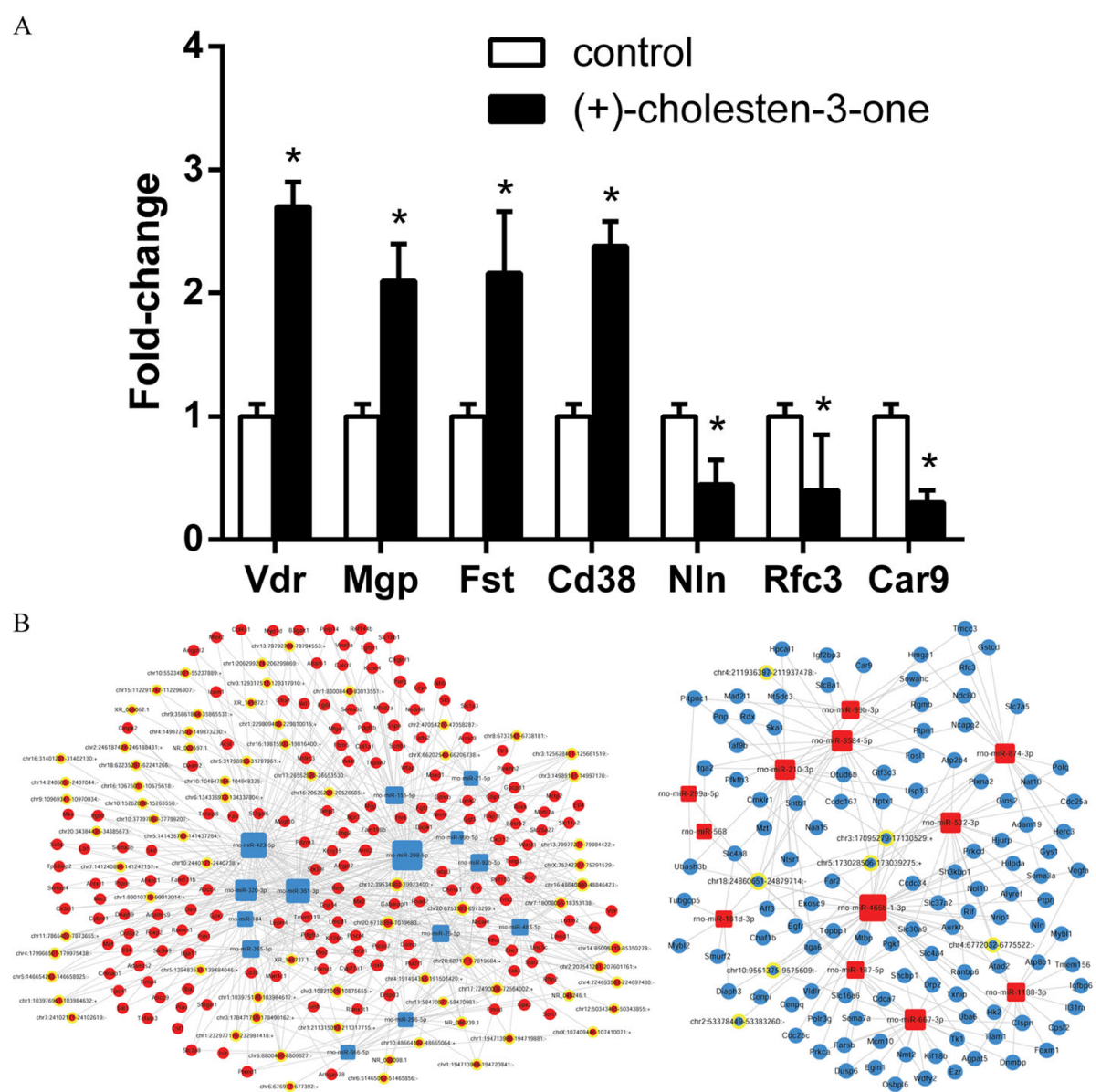

Fig. 2 Candidate mRNA predictions and CNC network construction during CN-induced MSC osteoblast differentiation. a In total, 443 mRNAs were predicted via mRBase targets based on the miRNA and IncRNA microarray. Seven mRNAs (Vdr, Mgp, Fst, Cd38, Nln, Rfc3 and Car9) were selected for validation via qRT-PCR, and the results were consistent with the predicted target genes. b CNC networks during the CN-induced osteoblast differentiation of MSCs. The dots represent mRNAs, the dots in circles represent IncRNAs, and the squares represent miRNAs. All the red dots represent upregulation, and all the blue dots represents downregulation. $P<0.05$, compared to the control group 
We then constructed a CNC network to study the interactions between miRNAs, mRNAs and lncRNAs that were differentially expressed during $\mathrm{CN}$-induced MSC osteoblast differentiation. The $\mathrm{CNC}$ network was based on normalized signal intensity and plotted using Cytoscape 3.0 (Fig. 2b). For visual analysis, the Pearson correlation coefficient was calculated first, and only the strongest correlations were included (Pearson correlation coefficient $>0.9$ ). A node represented a gene, and the edges between two nodes represented the connection between two genes. Degrees were defined as the number of directly related neighbours. Then, the relationship among miRNAsmRNAs-lncRNAs was integrated into the CNC network based on negative regulation. Finally, we identified 277 target mRNAs according to the degree values; of these, 165 were upregulated, and 112 were downregulated. Each mRNA corresponds to one or more miRNA and lncRNA and vice versa (Additional file 3).
Bioinformatic analysis of candidate genes during $\mathrm{CN}$-induced MSC osteoblast differentiation

First, gene ontology (GO) cluster analysis was conducted to further explore the biological processes involving the 277 candidate mRNAs. As a result, 174 biological processes were enriched, including 113 that were upregulated and 61 that were downregulated (Additional file 4). Between the upregulated biological processes, the response to mechanical stimulus, positive regulation of cell migration, positive regulation of cell proliferation, vitamin $\mathrm{D}$ receptor signalling pathway, and collagen fibril organization processes may be related to $\mathrm{CN}$-induced MSC osteoblast differentiation. Among the downregulated biological processes, the peptidyl-tyrosine dephosphorylation, positive regulation of protein localization to early endosome, cell proliferation, positive regulation of cell adhesion, and response to hypoxia processes may be related to $\mathrm{CN}$-induced

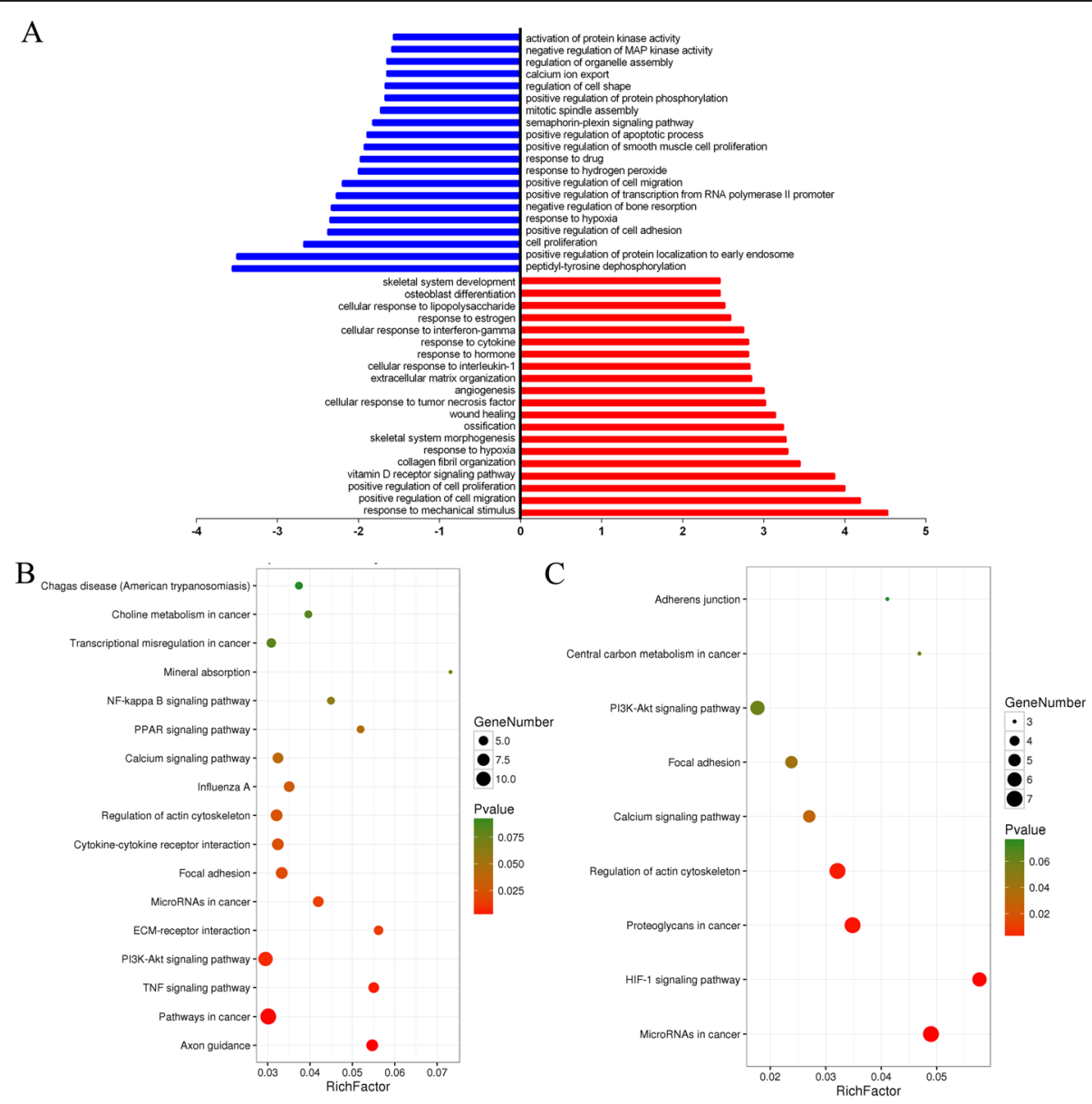

Fig. $3 \mathrm{GO}$ and KEGG analyses of predicted target genes during CN-induced MSC osteoblast differentiation. a GO analysis of 443 predicted target genes. In total, 174 biological processes were enriched; upregulation is shown in red, and downregulation is shown in blue. The y-axis shows the top ten biological processes, and the x-axis shows the negative logarithm of the $p$ value (-LgP) of each biological process. $\mathbf{b}$ KEGG analysis of upregulated predicted target genes. c KEGG pathway analysis of downregulated predicted target genes. In both B \& C, the $y$-axis shows the pathway category, and the $x$-axis shows the richness factor. A larger richness factor indicates greater enrichment. The size of the bubble indicates the number of genes in the pathway, and the colour of the bubble represents the range of $P$ values 
MSC osteoblast differentiation (Fig. 3a; Additional file 4). In addition, 59 upregulated genes and 45 downregulated genes were identified by GO cluster analysis.

Next, Kyoto Encyclopedia of Genes and Genomes(KEGG) pathway analysis was conducted to further explore the signalling pathways involving the 277 candidate mRNAs. In total, 26 significant signalling pathways related to $\mathrm{CN}$-induced MSC osteogenic differentiation were enriched, including 17 upregulated pathways and 9 downregulated pathways (Additional file 5). Among the upregulated pathways, the axon guidance, TNF signalling, PI3K-Akt signalling, calcium signalling, and mineral absorption pathways may be related to $\mathrm{CN}$-induced MSC osteoblast differentiation. Among the downregulated pathways, the microRNAs in cancer, HIF-1 signalling, proteoglycans in cancer, regulation of actin cytoskeleton, and focal adhesion pathways may be related to $\mathrm{CN}$-induced MSC osteoblast differentiation (Fig. 3b \& c; Additional file 5). In addition, 49 upregulated genes and 21 downregulated genes were identified by KEGG pathway analysis.

\section{Core CNC networks during CN-induced MSC osteoblast differentiation}

The core $\mathrm{CNC}$ network were then constructed based on the core genes(29 mRNAs), which were obtained from the same genes from GO and KEGG analyses (Fig. 4a; Additional file 6). The osteogenesis-related genes were identified using online databases (https://geneticassocia tiondb.nih.gov/, https://www.ncbi.nlm.nih.gov/gene/) and our core CNC networks. At last 6 core genes (Vdr, Mgp, Fabp3, Fst, Cd38, and Col1a1) were identified, and 5 core miRNAs (miR-483, miR-298, miR-361, miR-92b and miR-155) and 5 core lncRNAs (NR_046246.1, NR_046239.1, XR_086062.1, XR_145872.1 and XR_146737.1) were correspondingly identified (Fig. 4b; Additional file 7).

To confirm the reliability of the sub-core CNC networks and the online databases, the 5 core miRNAs, 5 core lncRNAs and 6 core mRNAs were verified via qRT-PCR and western blot analysis. The qRT-PCR (Fig. 4c, d \& e) and western blot (Fig. 4f \& g) results confirmed that the expression levels of these 16 core genes were consistent with the sub-core $\mathrm{CNC}$ networks and online databases.

\section{miR-298 directly targets Vdr during the $\mathrm{CN}$-induced osteoblast differentiation of MSCs}

To gain insight into the molecular mechanisms by which miR-298 regulates $\mathrm{CN}$-induced MSC osteogenic differentiation, we found that the osteogenic-specific gene vitamin D receptor (Vdr) has a miR-298 binding site in its 3'UTR. To test whether miR-298 directly targets Vdr, we constructed luciferase reporters that had either a wild-type 3'UTR (Vdr-3'UTR-wt) or a 3'UTR containing mutant (Vdr-3'UTR-mut) sequences of the miR-298 binding site. Overexpression of miR-298 remarkably inhibited the luciferase activity of Vdr-3'UTR-wt but not that of Vdr-3'UTR-mut (Fig. 5a \& b). These findings indicated that miR-298 may directly regulate Vdr expression. Western blot analysis confirmed that miR-298 overexpression markedly suppressed the expression of Vdr, Cd38 and Colla1 at the protein level (Fig. 5c \& d). These results indicate that $\mathrm{Vdr}$ is a miR-298 target gene.

miR-298 negatively regulates the expression of osteogenesisrelated factors

To evaluate the role of miR-298 in the osteoblast differentiation of MSCs, we transfected MSCs with a miR control, mimic miR-298 mimic or miR-298 inhibitor construct. Then, the cells were cultured with $\mathrm{CN}$ for 7 days to induce osteogenic differentiation. The alizarin red staining (AR-S) assay showed that miR-298 significantly suppressed MSC osteogenic differentiation compared with that of cells transfected with the miR control. However, miR-298 inhibitor markedly promoted osteogenic differentiation (Fig. 6a). The mRNA and protein levels of osteogenesis-related factors, such as alkaline phosphatase (ALP), osteopontin (OPN) and runt-related transcription factor 2 (RUNX2), were analysed by qRT-PCR and western blot after miR-298 mimic transfection. The qRT-PCR results showed that the mRNA levels of the osteogenic-related factors were decreased in the miR-298 mimic group compared with those in the miR control group, whereas these levels were enhanced in the miR-298 inhibitor group (Fig. 6b). Western blot analysis also demonstrated changes in the protein levels of these osteogenesis-related factors, which were similar to the changes in the mRNA levels (Fig. 6c \& d). These data suggest that miR-298 is the core miRNA functioning during the $\mathrm{CN}$-induced osteoblast differentiation of MSCs.

\section{Vdr overexpression eliminated the inhibitory effect of miR-298 during $\mathrm{CN}$-induced MSC osteoblast differentiation}

To further understand the functional effect of miR-298 on osteogenesis-related factors, such as ALP, OPN and RUNX2, by regulating Vdr, we transfected MSCs with miR-298 mimic and pcDNA 3.1-Vdr under CN induction. The AR-S assay showed that more calcium mineral deposition was observed in the pcDNA 3.1-Vdr transfected group than in the untransfected control and co-transfected pcDNA $3.1-\mathrm{Vdr}$ and miR-298 mimic groups (Fig. 7a). Furthermore, western blot analysis demonstrated that pcDNA 3.1-Vdr remarkably increased the expression of ALP, OPN and RUNX2. In addition, pcDNA $3.1-\mathrm{Vdr}$ significantly reversed the inhibitory effects of miR-298 overexpression on osteogenic differentiation, restoring the expression of the osteogenic-related factors ALP, OPN and RUNX2 (Fig. 7b \& c). These results 


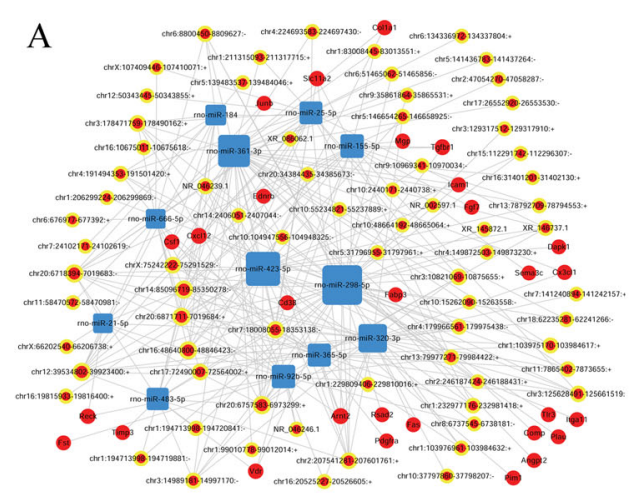

D

B
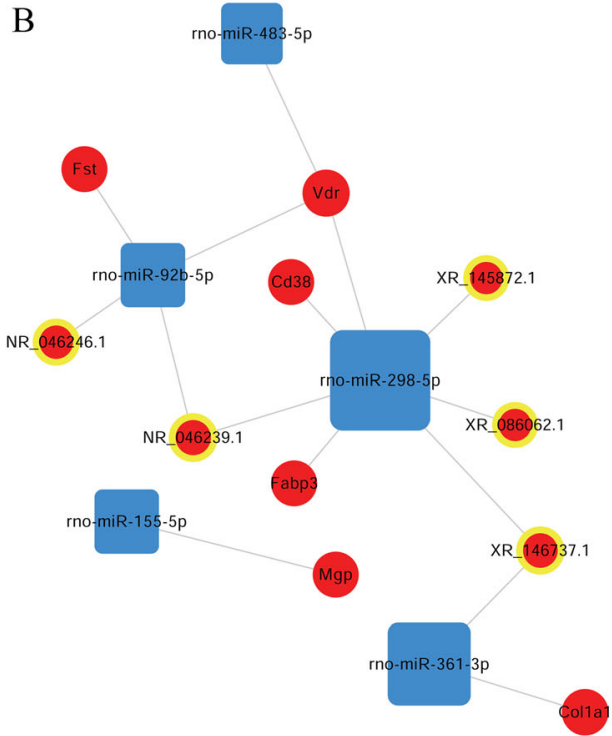

$\mathrm{C}$

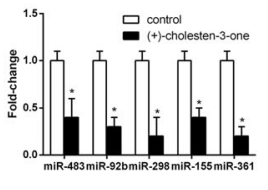

E

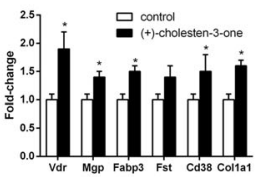

G

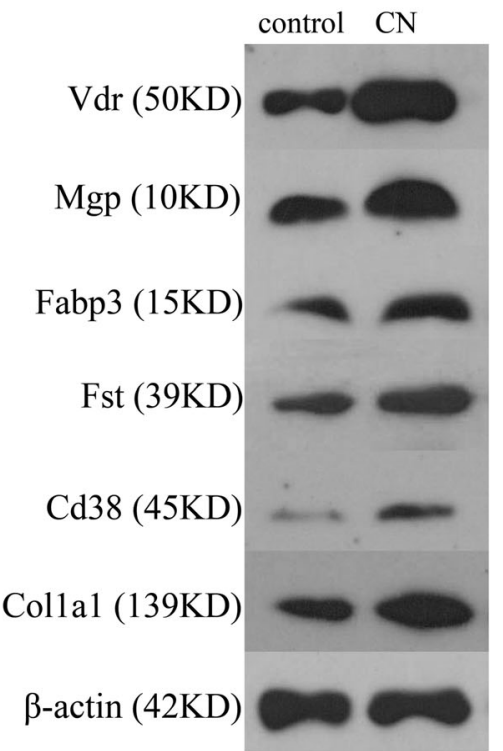

Fig. 4 Core CNC networks during the CN-induced osteoblast differentiation of MSCs. a Core CNC network based on the 29 core genes obtained from GO cluster analysis and KEGG pathway analysis. b Sub-core CNC networks based on the 6 core genes obtained from online databases and core CNC networks. c qRT-PCR verification of miRNA expression. Compared with the control group, the expression levels of all the core miRNAs were lower in the CN-induced MSC group, which was consistent with the sub-core CNC network results. $\mathbf{d}$ qRT-PCR verification of IncRNA expression. Compared with the control group, the expression levels of all the core IncRNAs were higher in the CN-induced MSC group, which was consistent with the sub-core CNC network results. e qRT-PCR verification of mRNA expression. Compared with the control group, the expression levels of all the core mRNAs were higher in the CN-induced MSC group, which was consistent with the sub-core CNC network results. $\mathbf{f}$ and $\mathbf{g}$ Western blot verification of mRNA expression; the results were similar to the qRT-PCR results. $P<0.05$, compared to the control group

indicate that $\mathrm{Vdr}$ overexpression abolished the inhibitory effect of miR-298 during the $\mathrm{CN}$-induced osteoblast differentiation of MSCs.

\section{Discussion}

The osteogenic differentiation of MSCs is precisely regulated by intrinsic molecular signals and the external environment [1]. Understanding the molecular mechanisms underlying MSC osteogenic differentiation is important for therapeutic purposes. Our previous reports $[5,6]$ indicated that $\mathrm{CN}$ has the potential to promote the osteogenic differentiation of MSCs. However, the role of epigenetic regulation in the $\mathrm{CN}$-induced osteogenic differentiation of MSCs remains poorly understood. Recently, miRNAs have emerged as important regulators of the osteoblast differentiation of MSCs. Several studies [7, 23-25] have reported that miRNAs target the critical transcription factors involved in the osteoblast differentiation of MSCs. Growing reports have shown that a number of key signalling pathways are involved in the regulation of osteoblast differentiation, including the TGF- $\beta /$ BMP signalling [22, 26], Wnt signalling [26, 27], Hedgehog signalling [28], Notch signalling $[29,30]$ and FGF signalling pathway [31]. Emerging evidence [32, 33] has revealed that lncRNAs may also play a vital role in regulating MSC osteogenic differentiation. For example, the regulation of miRNA-mRNA, IncRNA-mRNA, miRNA-lncRNA and miRNA-mRNA-IncRNA interactions has been reported in MSC osteogenic differentiation [21, 22]. However, the role of miRNA-mRNA-lncRNA interaction networks in the 


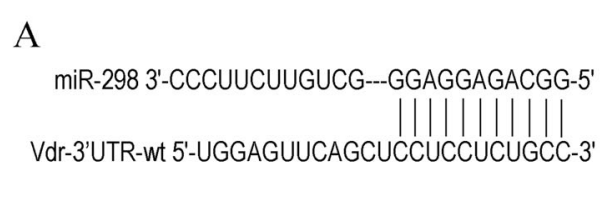

B

Vdr-3'UTR-mut 5'-UGGAGUUCAGCUAAGAAGAGUAA-3'
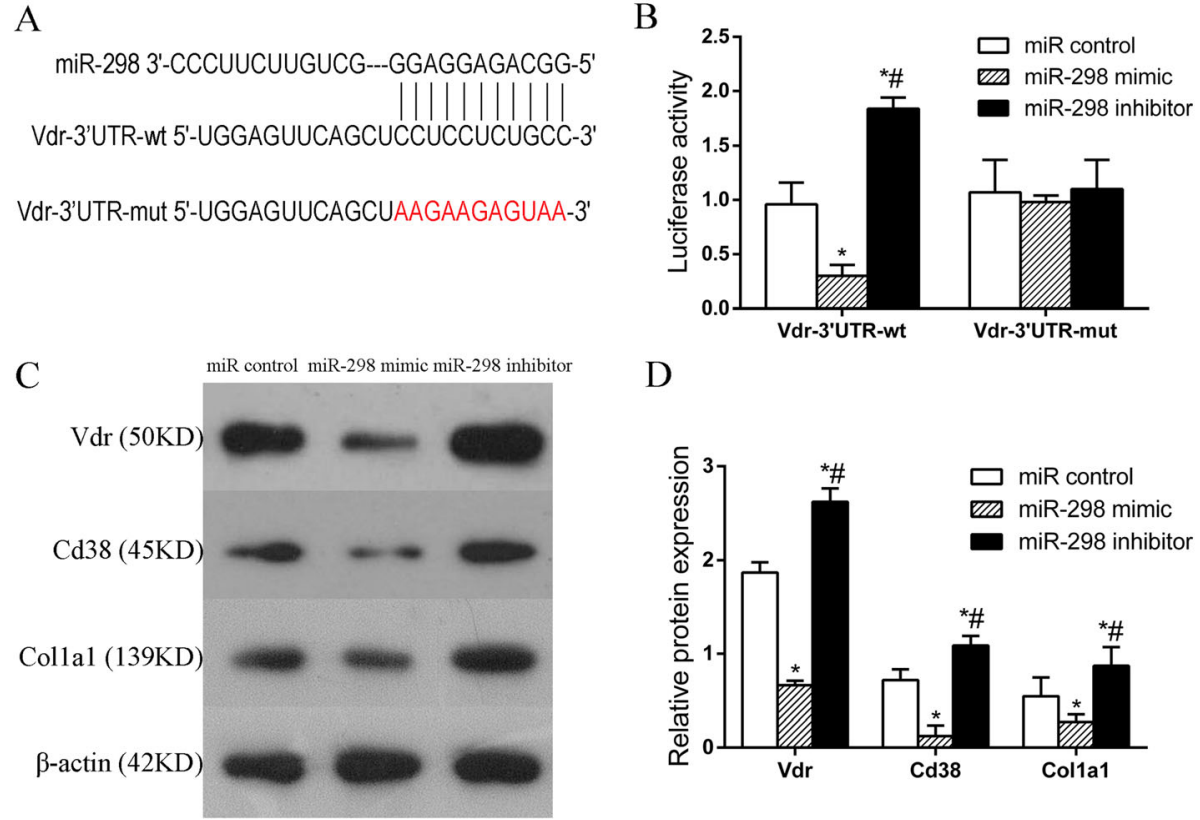

$\mathrm{D}$

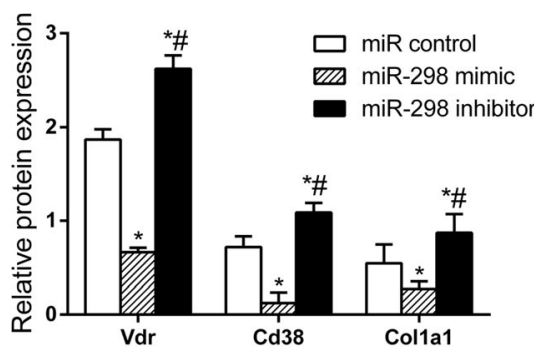

Fig. 5 miR-298 directly targets Vdr during CN-induced MSC osteoblast differentiation. a Predicted miR-298 target sequence in the $3^{\prime} U T R$ of Vdr. $\mathbf{b}$ Luciferase reporter assay of MSCs co-transfected with wt or mut Vdr plasmids and miR control, miR-298 mimic or miR-298 inhibitor, which showed that overexpression of miR-298 remarkably inhibited the luciferase activity of the wild-type Vdr 3'UTR (Vdr-3'UTR-wt) construct but not that of the mutated Vdr 3'UTR (Vdr-3'UTR-mut) construct. c and $\mathbf{d}$ Western blot detection of Vdr, Cd38, and Col1a1 protein expression in MSCs transfected with miR-298 mimic or miR-298 inhibitor. miR control group: MSCs transfected with miR control; miR-298 mimic group: MSCs transfected with miR-298 mimic; miR-298 inhibitor group: MSCs transfected with miR-298 inhibitor. ${ }^{*} P<0.05$ vs. the miR control group. \#P< 0.05 vs. the miR-298 mimic group

$\mathrm{CN}$-induced osteogenic differentiation of MSCs remains largely unknown.

In this study, we outlined the miRNA-mRNA-IncRNA interaction network during $\mathrm{CN}$-induced MSC osteogenic differentiation. Using miRNA and lncRNA microarray analysis, we identified 77 miRNAs (36 upregulated and 41 downregulated) and 295 differentially expressed lncRNAs, and 443 mRNAs were predicted based on the miRNA and lncRNA negative correlations. We then performed a dynamic analysis of the miRNA-mRNA-lncRNA interaction network during $\mathrm{CN}$-induced MSC osteogenic differentiation, which suggested that downregulated miRNAs, upregulated lncRNAs and mRNAs were predominant.

Further bioinformatic analysis was performed to screen the key genes controlling $\mathrm{CN}$-induced MSC osteogenic differentiation. First, GO cluster analysis identified 113 upregulated biological processes and 61 downregulated biological processes. Among the upregulated biological processes, the Vdr signalling pathway, response to mechanical stimulus, positive regulation of cell migration, positive regulation of cell proliferation, and collagen fibril organization processes, among others, may be related to $\mathrm{CN}$-induced MSC osteogenic differentiation. These results were also consistent with previous reports [34-39]. Among the downregulated biological processes, the positive regulation of protein localization to early endosomes, cell proliferation, positive regulation of cell adhesion, and response to hypoxia processes may be related to $\mathrm{CN}$-induced MSC osteogenic differentiation; these results were also consistent with those previously reported [4044]. KEGG analysis was then used to identify 26 signalling pathways significantly related to $\mathrm{CN}$-induced MSC osteogenic differentiation, including the axon guidance, TNF signalling, PI3K-Akt signalling, calcium signalling, mineral absorption, HIF-1 signalling, regulation of actin cytoskeleton, and focal adhesion pathways. Kim et al. used a fractionated secretomic approach and identified the axon guidance molecule SLIT3 as a clastokine that stimulates osteoblast migration and proliferation by activating $\beta$-catenin and confirmed that SLIT3 inhibits bone resorption by suppressing osteoclast differentiation in an autocrine manner [45]. Bai et al. found that TNF- $\alpha$ inhibits osteoblast differentiation mainly by activating the nuclear factor (NF)- $\mathrm{kB}$ signalling pathway [46]. Using in vivo and in vitro analyses, $\mathrm{Xi}$ et al. indicated that the PI3K/Akt cell signalling pathway is involved in osteoporosis inhibition by promoting osteoblast proliferation, differentiation and bone formation [47]. The calcium signalling pathway and mineral absorption have also been reported previously $[48,49]$.

Bioinformatic analysis of the miRNA-mRNA-lncRNA interaction network identified 16 core genes, including 6 mRNAs (Vdr, Mgp, Fabp3, Fst, Cd38, and Col1a1), 5 miRNAs (miR-483, miR-298, miR-361, miR-92b and 
A

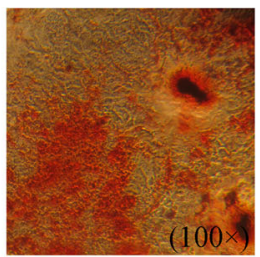

miR control

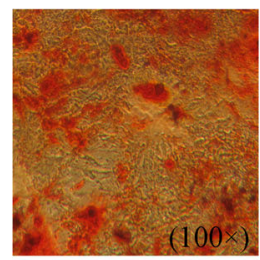

miR-298 mimic

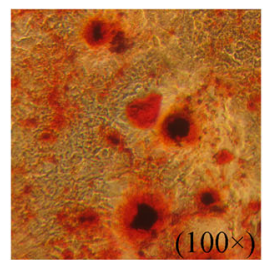

miR-298 inhibitor

B
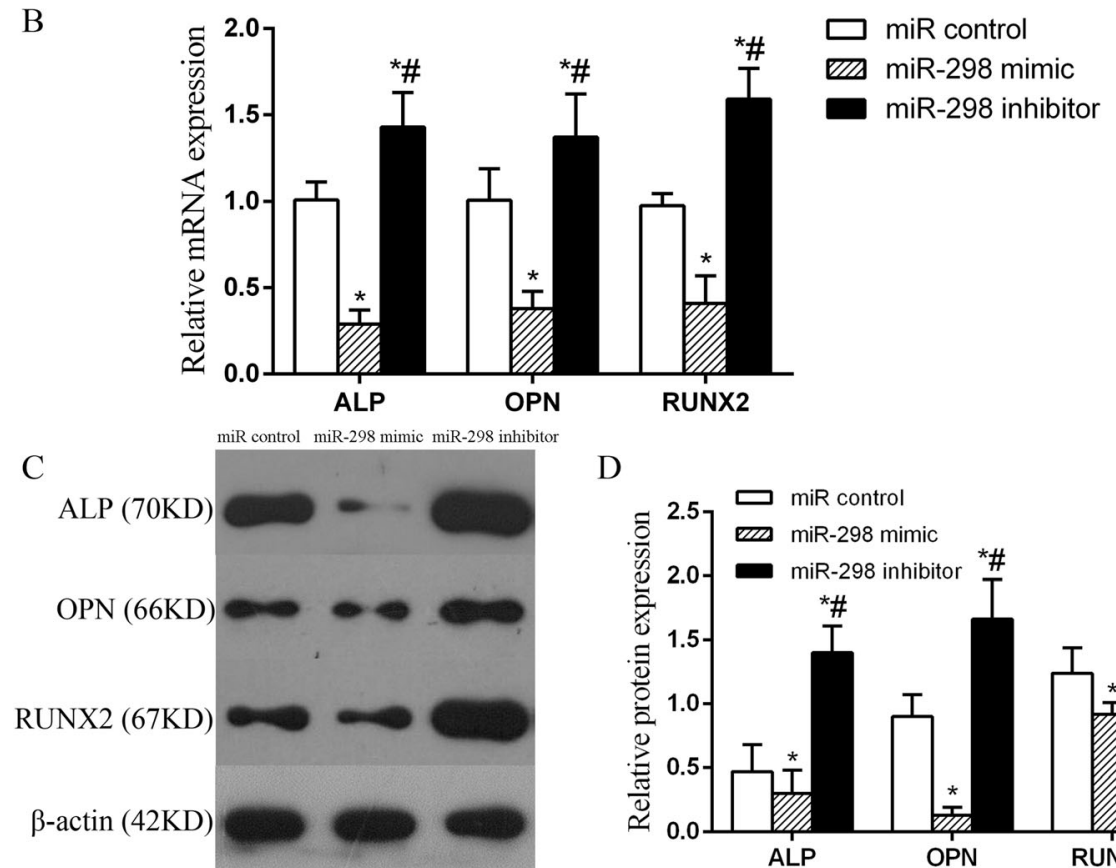

$\mathrm{D}$

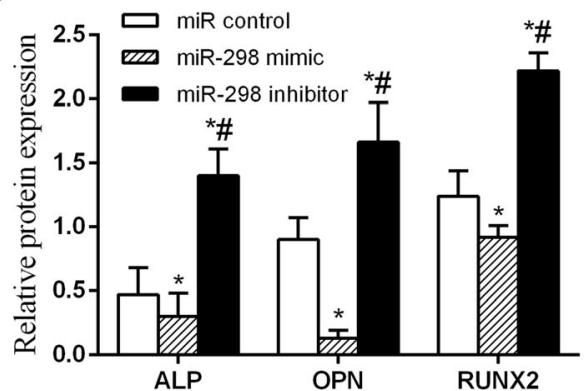

Fig. 6 miR-298 negatively regulated osteogenesis-related factors during CN-induced MSC osteoblast differentiation. a AR-S showed that more calcium mineral deposition was found in the miR-298 inhibitor group than in the miR control and miR-298 mimic groups; in other words, miR-298 suppressed MSC osteogenic differentiation. $\mathbf{b}$ qRT-PCR detection of the mRNA expression of osteogenic-related factors in MSCs transfected with miR-298 mimic or miR-298 inhibitor showing that the mRNA levels of the osteogenic-related factors were decreased in the miR-298 mimic group compared with those in the miR control group, whereas these levels were enhanced in the miR-298 inhibitor group. $\mathbf{c}$ and $\mathbf{d}$ Western blot detection of the protein expression of osteogenic-related factors in MSCs transfected with miR-298 mimic or miR-298 inhibitor; the results were similar to the qRT-PCR results. miR control group: MSCs transfected with miR control; miR-298 mimic group: MSCs transfected with miR-298 mimic; miR-298 inhibitor group: MSCs transfected with miR-298 inhibitor. ${ }^{*} P<0.05$ vs. the miR control group. $\# P<0.05$ vs. the miR-298 mimic group

miR-155), and 5 lncRNAs (NR_046246.1, NR_046239.1, XR_086062.1, XR_145872.1 and XR_146737.1). Vdr, a ligand-activated transcription factor, has been reported to be a core gene during MSC osteogenic differentiation [5, $6,50]$, and matrix gla protein (Mgp) has been recognized as a potent calcification inhibitor and regulator of bone morphogenetic protein-2 (BMP-2) [51]. Wang et al. [52] indicated that overexpression of fatty acid binding protein 3 (Fabp3) inhibited MSC growth and proliferation via negatively regulating the cell cycle and MSC growth factors and enhancing cell survival under hypoxic or ischaemic conditions, which indicated that the Fabp3 gene potentially promotes MSC osteogenic differentiation. Gajos-Michniewicz et al. indicated that overexpression of follistatin (Fst) negatively influences bone metabolism and induces a significant decrease in biomechanical strength variables in FST-overexpressing mice compared to those in control mice. Overexpression of FST leads to decreased skeletal quality, thus increasing susceptibility to bone fractures [53], and Cd38 expression may increase the intracellular calcium concentration [54]. Col1a1, a marker of osteogenic differentiation, shows higher expression levels during the osteoblast differentiation of bone marrow stromal cells throughout osteogenesis [55]. The reliabilities of the sub-core $\mathrm{CNC}$ networks and online databases were verified via $\mathrm{qRT}$-PCR and western blot.

A high degree of enrichment means that enrichment plays an important role in regulating $\mathrm{CN}$-induced MSC osteogenic differentiation. Among the core miRNAs, mRNAs and lncRNAs, miR298 was the most significantly downregulated and associated with 6 enriched genes, while $\mathrm{Vdr}$ was associated with 3 enriched genes. 

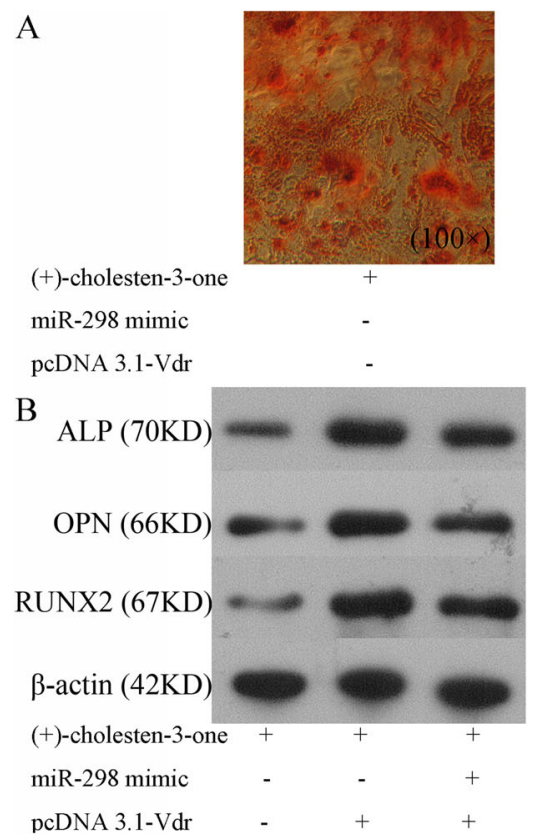
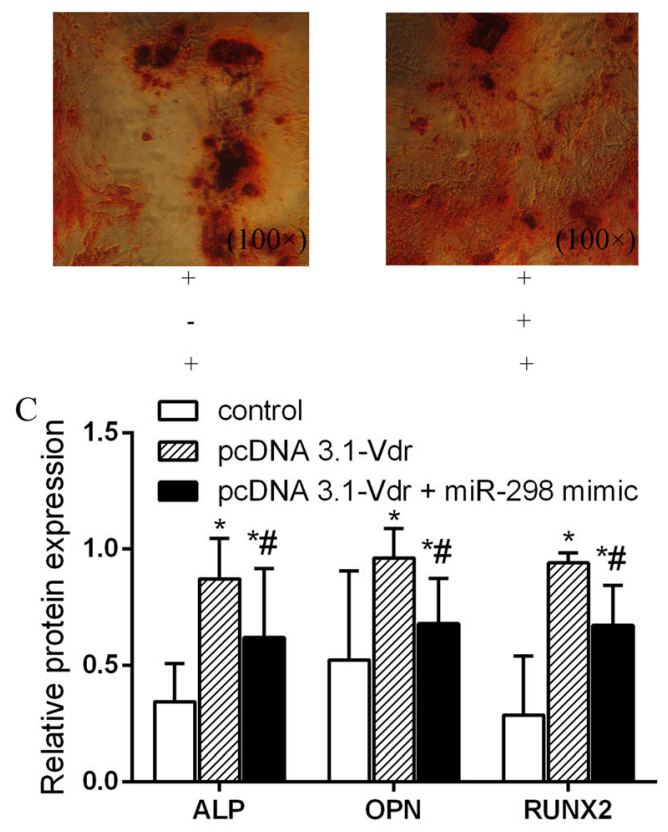

Fig. $7 \mathrm{Vdr}$ overexpression rescues the inhibitory effect of miR-298 during the CN-induced osteoblast differentiation of MSCs. a AR-S showed that more calcium mineral deposition was found in the CN + pCDNA 3.1-Vdr + miR-298 mimic group than in the CN group, while less calcium mineral deposition was found in the CN + pcDNA 3.1-Vdr group. Thus, pcDNA 3.1-Vdr rescued the inhibitory effect of miR-298 during the CN-induced osteoblast differentiation of MSCs. $\mathbf{b}$ \& $\mathbf{c}$ Western blot detection of the protein expression of osteogenic-related factors in MSCs transfected with miR-298 mimic or pcDNA 3.1-Vdr; the results were similar to the AR-S results. Control group: MSCs transfected without pcDNA 3.1-Vdr or miR-298 mimic; pcDNA 3.1-Vdr group: MSCs transfected with pcDNA 3.1-Vdr; pcDNA 3.1-Vdr + miR-298 mimic group: MSCs co-transfected with pcDNA 3.1-Vdr and miR-298 mimic. ${ }^{*} P<0.05$ vs. the $C N$ group. $\# P<0.05$ vs. the miR-298 mimic group

Subsequent bioinformatics analysis revealed that miR-298 interacted closely with Vdr. Coincidentally, our previous study showed that Vdr positively regulates osteoblast differentiation induced by $\mathrm{CN}[5,6]$. We then further examined the function of miR-298 in MSC osteogenic differentiation using transfection methods and found that miR-298 significantly inhibited MSC osteogenic differentiation. miR-298 upregulation decreased osteoblast differentiation by inhibiting Vdr in MSCs. Following osteoblast differentiation induced by CN, miR-298 was significantly downregulated, and Vdr was no longer inhibited by miR-298, thereby facilitating the escape of MSCs from the quiescent state into osteogenic differentiation. However, how Vdr and miR-298 precisely regulate the osteogenic differentiation of MSCs requires further study. Nonetheless, the above results provide sufficient evidence that $\mathrm{Vdr}$ is a predicted miR-298 target gene. Bioinformatics analysis and a luciferase reporter assay verified that miR-298 inhibited the expression of $\mathrm{Vdr}$ via directly binding the 3'UTR region of its mRNA. Overexpression of Vdr markedly reversed the inhibitory effects of miR-298, indicating that Vdr was the effector of miR-298 during MSC osteogenic differentiation. Taken together, these results indicate that miR-298 may be a key factor in inhibiting MSC osteogenic differentiation and that $\mathrm{Vdr}$ is a miR-298 target gene.
Our data also showed that the expression levels of NR_046246.1, NR_046239.1, XR_086062.1，XR_145872.1 and XR_146737.1 were increased, while those of miR-483, miR-298, miR-361, miR-92b and miR-155 were decreased during $\mathrm{CN}$-induced MSC osteogenic differentiation. Because miR-298 performed outstandingly in both the microarray and bioinformatics analyses, we treated it as the centre of our network. We used miRDE (http://www.mirdb.org), starBasehttp://starbase.sysu.edu.cn, and Segal Lab of Computational Biology (https://genie.weizmann.ac.il/pubs/ mir07/mir07_prediction.html) to predict the binding sites between miR-298 and the other core IncRNAs, but no unequivocal relationships were observed. While none of the core lncRNAs have been reported previously, they may act as "sponges" to other miRNAs that we did not evaluate in this study, and we will conduct further examinations to verify their roles in the network. The lncRNAs KCNQ1OT1 [56], MALAT [57], XR_111050 [22], etc. reportedly promote osteogenic differentiation, while the lncRNAs HOTAIR [58], DANCR [59], TSIX [60], etc. reportedly inhibit osteogenic differentiation. miR-483-5p is overexpressed in osteoporotic patients and targets the downregulation of IGF2 expression [61], while miR-92b promotes cell proliferation and invasion in osteosarcoma by targeting DKK3 [62], and miR-155 inhibits 
osteoblast differentiation by downregulating the translation of SMAD5 in mouse pre-osteoblasts [63].

\section{Conclusions}

We dynamically analysed the interaction network of coding and noncoding transcripts in $\mathrm{CN}$-induced MSC osteogenic differentiation. Further bioinformatic analyses and in vitro experiments confirmed that miR-298 is required for $\mathrm{CN}$-induced MSC osteogenic differentiation. Our study laid the foundation for further understanding the exact mechanism underlying the miRNA-mRNA-lncRNA interaction network regulating MSC osteogenic differentiation. In addition, the miRNAs and lncRNAs that are differentially expressed during MSC osteogenic differentiation may be potential targets for bone tissue engineering.

\section{Methods}

\section{Isolation, culture and $\mathrm{CN}$-induced MSC osteoblast differentiation}

Sprague dawley rats were used in this study and euthanised by cervical dislocation. MSCs were isolated and cultured as described in our previous reports $[5,6]$ (Supplement 1). The initial culture medium was then replaced with fresh medium supplemented with $30 \mu \mathrm{g} /$ $\mathrm{mL} \mathrm{CN}, 10 \% \mathrm{FBS}$, and $1 \%$ penicillin-streptomycin to induce MSC osteoblast differentiation for 7 days and refreshed every 3 days thereafter.

\section{miRNA and IncRNA microarray analysis}

After $\mathrm{CN}$-induced osteoblast differentiation, MSCs were subjected to miRNA and lncRNA microarray assays performed by RiboBio Co., Ltd. (Guangzhou, China). The method was the same as that used in our previous report [6] (Supplement 2).

Quantitative real-time polymerase chain reaction (qRT-PCR) qRT-PCR was used to verify the expression of miRNAs, lncRNAs and mRNAs and to detect the expression of osteogenic differentiation markers as described in our previous report [6] (Supplement 3). Primer sequences are listed in Additional file 8.

\section{Candidate mRNA prediction}

Candidate mRNAs targeted by the differentially expressed miRNAs identified in this study were predicted by Genminix Informatics Ltd., Co. (Shanghai, China) using miRanda (http://miranda.org.uk/). The miRanda method is based on dynamic programming (Smith-Waterman (SW) algorithm [64]) and the computation of free energy. When the sequence alignment score value (miRNA and 3'-UTR) and free energy are greater than the corresponding pre-defined thresholds, the gene for which the 3'-UTR is a sub-sequence is considered the target gene of the miRNA. During this process, the concrete alignment is obtained.
During alignment, every pair of bases has a score value (may be negative), and the ultimate score is the sum of all the values. The matched score value is greater than the mismatched score value, and the term wobble is used when the mismatched pair, T and G, has a greater score value than the other mismatched pairs. Insertions or deletions may occur during alignment, which are represented by "-". Every gap has a penalty (negative sore value), and open gaps have a smaller penalty than extensive gaps. miRanda defines seeds, but gaps at seeds are not allowed, and positive score values and penalty score values occur several times more often in seeds than in non-seed areas.

\section{Construction of the CNC network}

The CNC network was built according to the normalized signal intensity of miRNAs, lncRNAs and mRNAs. We calculated the Pearson correlation coefficient between two genes, and only the strong correlations $(>0.9)$ were selected to construct the CNC network, which was drawn with Cytoscape 3.0. Construction and visualization of the network were performed by Genminix Informatics Ltd., Co. (Shanghai, China).

\section{GO and KEGG analyses}

GO and KEGG analyses were performed to identify the biological processes, molecular functions, cellular components and pathways as previously described [21] (Supplement 4).

\section{Core CNC networks}

The core $\mathrm{CNC}$ network were then constructed based on the core genes(29 mRNAs), which were obtained from the same genes from GO and KEGG analyses. Two online databases (https://geneticassociationdb.nih.gov/, https:// www.ncbi.nlm.nih.gov/gene/) were used to identify the osteogenic differentiation-related genes in the core $\mathrm{CNC}$ networks. The core genes identified from the core $\mathrm{CNC}$ networks and online databases were verified by qRT-PCR and western blotting [22].

\section{Bioinformatic analysis}

miRanda (http://www.microrna.org), PicTar (http://pic tar.mdc-berlin.de) and targetScan (http://www.targetsca n.org) were used to analyse potential miR-298 binding sites on the Vdr 3'-UTR. The consistency of the analyses by these three websites suggested that the results were reliable, and the results were further verified using in vitro experiments.

\section{Luciferase reporter assays and MSC transfection with miR-298 mimic, miR-298 inhibitor and pcDNA 3.1-Vdr} Luciferase reporter assays were performed to identify whether miR-298 directly targets $\mathrm{Vdr}$ expression, as 
described in our previous study [6] (Supplement 5). MSCs transfected with miR-298 mimic, miR-298 inhibitor, or Vdr-overexpressing vector(pcDNA 3.1-Vdr, a Vdr recombinant vectors without 3 'UTR) were subsequently subjected to AR-S and western blot analyses to clarify the role of miR-298 in MSC osteogenic differentiation as described in our previous report [6] (Supplement 6).

\section{Calcium mineral deposition}

After transfection with a miR-298 mimic, miR-298 inhibitor or Vdr-overexpressing vector, MSCs continued to differentiate into osteoblast via $\mathrm{CN}$ for 14 days. AR-S was used to detect the osteogenic differentiation ability mediated by miR-298 and pcDNA 3.1-Vdr as described in our previous report [6] (Supplement 7).

\section{Western blot}

Osteogenic-specific factors, such as ALP, OPN and RUNX2 were detected by western blot after the transfection of MSCs with miR-298 mimic, inhibitor and pcDNA 3.1-Vdr as described in our previous report [6] (Supplement 8).

\section{Statistical analysis}

Statistical analyses were performed using SPSS 19.0 (SPSS Inc., Chicago, IL, USA). Statistical analyses included the t-test, Fisher's exact test, $x^{2}$ test, one-way analysis of variance (ANOVA), least significant difference (LSD) and Pearson correlation based on the data type. $P<0.05$ was considered statistically significant.

\section{Additional files}

\section{Additional file 1: miRNA and IncRNA microarray of MSC osteoblast} differentiation induced by CN. (XLS $21 \mathrm{~kb}$ )

Additional file 2: Target gene prediction via mRBase targets based on the miRNA and IncRNA microarray. (XLS $155 \mathrm{~kb}$ )

Additional file 3: Relationship between miRNAs, mRNAs and IncRNAs according to the negative regulation. (XLSX $144 \mathrm{~kb}$ )

Additional file 4: $\mathrm{GO}$ analysis of target genes obtained from CNC networks. (XLSX 52 kb)

Additional file 5: KEGG pathway analysis of target genes obtained from CNC networks. (XLSX $31 \mathrm{~kb}$ )

Additional file 6: The core CNC networks were analysed based on the 29 mRNAs obtained from GO and KEGG pathway analyses. (XLSX 13 kb)

Additional file 7: Core CNC network analyses based on the online databases. (XLSX $42 \mathrm{~kb}$ )

Additional file 8: Primer sequences used for qRT-PCR. (XLSX 9 kb)

Additional file 9: Supplementary Materials. (DOC 37 kb)

\section{Abbreviations}

CN: (+)-cholesten-3-one; MSCs: Mesenchymal stem cells; miRNAs: MicroRNAs; InCRNAs: Long-chain noncoding RNAs; CNC: Coding-noncoding gene coexpression; GO: Gene ontology; KEGG: Kyoto Encyclopedia of Genes and Genomes; Vdr: Vitamin D receptor; AR-S: Alizarin red staining; ALP: Alkaline phosphatase; OPN: Osteopontin; RUNX2: Runt-related transcription factor 2; Mgp: Matrix gla protein; BMP-2: Bone morphogenetic protein-2; Fabp3: Fatty acid binding protein 3; qRT-PCR: Quantitative real-time polymerase chain reaction; ANOVA: One-way analysis of variance; LSD: Least significant difference

\section{Acknowledgements}

We thank all the people who contributed to the present study. We also thank Genminix Informatics Ltd., Co. (Shanghai, China) for their assistance with data processing.

\section{Ethical approval and consent to participate}

All animals received humane care in accordance with guidelines set by the Care of Experimental Animals Committee of Guangzhou University of Chinese Medicine and this study is consented by Guangzhou University of Chinese Medicine.

\section{Funding}

This study was supported by the Natural Science Foundation of Guangdong Province (no. 2017A030312009), the China Postdoctoral Science Foundation (no. 2017 M612641) and the Guangdong Provincial Traditional Chinese Medicine Research Project (no. 20181095, no. 20182043).

\section{Availability of data and materials}

The microarray datasets supporting the conclusions of this article are available. All other datasets supporting this article have been uploaded as part of the Additional file 9

\section{Authors' contributions}

$\mathrm{QH}, \mathrm{YH}$ and $\mathrm{DC}$ designed the research; $\mathrm{QH}, \mathrm{YH}, \mathrm{YL}, \mathrm{YL}, \mathrm{BW}, \mathrm{RD}$ and $\mathrm{SZ}$ performed the research; $\mathrm{YH}, \mathrm{YL}, \mathrm{FL}$ and $\mathrm{DC}$ analysed the data; $\mathrm{QH}$ and $\mathrm{DC}$ provided financial support; and $\mathrm{QH}$ and $\mathrm{YH}$ wrote the paper. All authors have read and approved the final manuscript.

\section{Consent for publication}

Not applicable.

\section{Competing interests}

The authors declare that they have no competing interests.

\section{Publisher's Note}

Springer Nature remains neutral with regard to jurisdictional claims in published maps and institutional affiliations.

\section{Author details}

${ }^{1}$ Department of Anatomy, The Research Centre of Integrative Medicine, Guangzhou University of Chinese Medicine, Guangzhou 510000, Guangdong, People's Republic of China. ${ }^{2}$ The First Affiliated Hospital of Guangzhou University of Chinese Medicine, Guangzhou 510000, Guangdong, People's Republic of China. ${ }^{3}$ Department of Orthopaedics, The Second Affiliated Hospital of Guangzhou University of Chinese Medicine, Guangzhou 510000 Guangdong, People's Republic of China. ${ }^{4}$ Department of Diagnosis of Traditional Chinese Medicine, Guangzhou University of Chinese Medicine Guangzhou 510000, Guangdong, People's Republic of China. ${ }^{5}$ Department of Trauma, The Third Affiliated Hospital of Guangzhou University of Chinese Medicine, Guangzhou 510000, Guangdong, People's Republic of China.

Received: 18 March 2018 Accepted: 10 October 2018

Published online: 29 October 2018

\section{References}

1. Bianco P, Riminucci M, Gronthos S, Robey PG. Bone marrow stromal stem cells: nature, biology, and potential applications. Stem Cells. 2001;19(3):180-92.

2. Bianco P, Robey PG, Simmons PJ. Mesenchymal stem cells: revisiting history, concepts, and assays. Cell Stem Cell. 2008:2(4):313-9.

3. Chen $Q$, Shou P, Zheng C, et al. Fate decision of mesenchymal stem cells: adipocytes or osteoblasts? Cell Death Differ. 2016;23(7):1128-39.

4. Deng P, Zhou C, Alvarez R, Hong C, Wang CY. Inhibition of IKK/NF-kappaB signaling enhances differentiation of mesenchymal stromal cells from human embryonic stem cells. Stem Cell Rep. 2016:6(4):456-65.

5. Hou QK, Huang YQ, Luo YW, et al. (+)-Cholesten-3-one induces osteogenic differentiation of bone marrow mesenchymal stem cells by activating vitamin D receptor. Exper Ther Med. 2017;13(5):1841-9. 
6. Hou Q, Huang Y, Luo Y, Wang B, Liu Y, Deng R, Zhang S, Liu F, Chen D. MiR-351 negatively regulates osteoblast differentiation of MSCs induced by (+)-cholesten3-one through targeting Vdr. Am J Transl Res. 2017;9(11):4963-73.

7. Heilmeier U, Hackl M, Skalicky S, Weilner S, Schroeder F, Vierlinger K, et al. Serum miRNA signatures are indicative of skeletal fractures in postmenopausal women with and without type 2 diabetes and influence osteogenic and Adipogenic differentiation of adipose tissue-derived mesenchymal stem cells in vitro. J Bone Miner Res. 2016;31(12):2173-92.

8. O'Hara SP, Mott JL, Splinter PL, Gores GJ, LaRusso NF. MicroRNAs: key modulators of posttranscriptional gene expression. Gastroenterology. 2009; 136:17-25.

9. Luo X, Dong Z, Chen Y, Yang L, Lai D. Enrichment of ovarian cancer stemlike cells is associated with epithelial to mesenchymal transition through an miRNA-activated AKT pathway. Cell Prolif. 2013;46(4):436-46.

10. Gothelf $Y$, Kaspi $H$, Abramov N, Aricha R. miRNA profiling of NurOwn(R): mesenchymal stem cells secreting neurotrophic factors. Stem Cell Res Ther. 2017;8(1):249.

11. Lytle JR, Yario TA, Steitz JA. Target mRNAs are repressed as efficiently by microRNA-binding sites in the 5' UTR as in the 3' UTR. Proc Natl Acad Sci U S A. 2007;104(23):9667-72.

12. Orom UA, Nielsen FC, Lund AH. MicroRNA-10a binds the 5'UTR of ribosomal protein mRNAs and enhances their translation. Mol Cell. 2008;30(4):460-71.

13. Iyer MK, Niknafs YS, Malik R, Singhal U, Sahu A, Hosono Y, et al. The landscape of long noncoding RNAs in the human transcriptome. Nat Genet. 2015;47(3): 199-208.

14. Necsulea A, Soumillon M, Warnefors M, Liechti A, Daish T, Zeller U, et al. The evolution of IncRNA repertoires and expression patterns in tetrapods. Nature. 2014;505(7485):635-40.

15. Guttman M, Amit I, Garber M, et al. Chromatin signature reveals over a thousand highly conserved large non-coding RNAs in mammals. Nature. 2009;458(7235):223-2277.

16. Yan $X$, Hu Z, Feng $Y$, et al. Comprehensive genomic characterization of long non-coding RNAs across human cancers. Cancer Cell. 2015;28(4):529-40.

17. Casero D, Sandoval S, Seet CS, et al. Long non-coding RNA profiling of human lymphoid progenitor cells reveals transcriptional divergence of $B$ cell and T cell lineages. Nat Immunol. 2015;16(12):1282-91.

18. Luo S, Lu JY, Liu L, Yin Y, Chen C, Han X, et al. Divergent IncRNAs regulate gene expression and lineage differentiation in pluripotent cells. Cell Stem Cell. 2016;18(5):637-52.

19. Lee S, Kopp F, Chang TC, Sataluri A, Chen B, Sivakumar S, et al. Noncoding RNA NORAD regulates genomic stability by sequestering PUMILIO proteins. Cell. 2016;164(1-2):69-80.

20. Zhou J, Yang L, Zhong T, Mueller M, Men Y, Zhang N, et al. H19 IncRNA alters DNA methylation genome wide by regulating $\mathrm{S}$ adenosylhomocysteine hydrolase. Nat Commun. 2015;6:10221.

21. Wu Q, Guo L, Jiang F, Li L, Li Z, Chen F. Analysis of the miRNA-mRNA-IncRNA networks in ER+ and ER- breast cancer cell lines. J Cell Mol Med. 2015;19(12): 2874-87.

22. Zhang W, Dong R, Diao S, Du J, Fan Z, Wang F. Differential long noncoding RNA/mRNA expression profiling and functional network analysis during osteogenic differentiation of human bone marrow mesenchymal stem cells. Stem Cell Res Ther. 2017;8(1):30.

23. Hu Z, Wang $Y$, Sun $Z$, Wang $H$, Zhou $H$, Zhang $L$, et al. miRNA-132-3p inhibits osteoblast differentiation by targeting Ep300 in simulated microgravity. Sci Rep. 2015;5:18655.

24. Le MH, Warotayanont R, Stahl J, Den Besten PK, Nakano Y. Amelogenin Exon4 forms a novel miRNA that directs Ameloblast and osteoblast differentiation. J Dent Res. 2016;95(4):423-9.

25. Suh JS, Lee JY, Choi YS, Chung CP, Park YJ. Peptide-mediated intracellular delivery of miRNA-29b for osteogenic stem cell differentiation. Biomaterials. 2013;34(17):4347-59

26. Wang T, Zhang X, Bikle DD. Osteogenic differentiation of periosteal cells during fracture healing. J Cell Physiol. 2017;232(5):913-21.

27. Zhang W, Xue D, Yin H, Wang S, Li C, Chen E, et al. Overexpression of HSPA1A enhances the osteogenic differentiation of bone marrow mesenchymal stem cells via activation of the Wnt/beta-catenin signaling pathway[]]. Sci Rep. 2016;6:27622.

28. Morsczeck C, Reck A, Beck HC. The hedgehog-signaling pathway is repressed during the osteogenic differentiation of dental follicle cells. Mol Cell Biochem. 2017;428(1-2):79-86.

29. Guo J, Fei C, Zhao Y, Zhao S, Zheng Q, Su J, et al. Lenalidomide restores the osteogenic differentiation of bone marrow mesenchymal stem cells from multiple myeloma patients via deactivating notch signaling pathway. Oncotarget. 2017;8(33):55405-21.

30. Yu GY, Zheng GZ, Chang B, Hu QX, Lin FX, Liu DZ, et al. Naringin Stimulates Osteogenic Differentiation of Rat Bone Marrow Stromal Cells via Activation of the Notch Signaling Pathway. Stem Cells Int. 2016;2016:7130653.

31. Simann M, Le Blanc S, Schneider V, Zehe V, Ludemann M, Schutze N, et al. Canonical FGFs prevent osteogenic lineage commitment and differentiation of human bone marrow stromal cells via ERK1/2 signaling. J Cell Biochem. 2017;118(2):263-75.

32. Huang $Y$, Zheng $Y$, Jia L, Li W. Long noncoding RNA H19 promotes osteoblast differentiation via TGF- $\beta 1 / \mathrm{Smad} 3 / \mathrm{HDAC}$ signaling pathway by deriving miR-675. Stem Cells. 2015;33(12):3481-92.

33. Zhuang W, Ge X, Yang S, Huang M, Zhuang W, Chen P, et al. Upregulation of IncRNA MEG3 promotes osteogenic differentiation of mesenchymal stem cells from multiple myeloma patients by targeting BMP4 transcription. Stem Cells. 2015;33(6):1985-97.

34. Doroudi M, Chen J, Boyan BD, Schwartz Z. New insights on membrane mediated effects of 1alpha, 25-dihydroxy vitamin D3 signaling in the musculoskeletal system. Steroids. 2014;81:81-7.

35. Pierroz DD, Bonnet N, Bianchi EN, et al. Deletion of beta-adrenergic receptor 1,2 , or both leads to different bone phenotypes and response to mechanical stimulation. J Bone Miner Res. 2012;27(6):1252-62.

36. Serrano JC, Cora-Cruz J, Diffoot-Carlo N, Sundaram PA. Adaptive responses of murine osteoblasts subjected to coupled mechanical stimuli. J Mech Behav Biomed Mater. 2018;77:250-7.

37. Horikiri $Y$, Shimo T, Kurio N, et al. Sonic hedgehog regulates osteoblast function by focal adhesion kinase signaling in the process of fracture healing. PLoS One. 2013;8(10):e76785

38. Uchihashi K, Aoki S, Matsunobu A, Toda S. Osteoblast migration into type I collagen gel and differentiation to osteocyte-like cells within a selfproduced mineralized matrix: a novel system for analyzing differentiation from osteoblast to osteocyte. Bone. 2013;52(1):102-10.

39. Burke M, Golaraei A, Atkins A, et al. Collagen fibril organization within rat vertebral bone modified with metastatic involvement. J Struct Biol. 2017; 199(2):153-64.

40. Solberg LB, Stang E, Brorson SH, Andersson G, Reinholt FP. Tartrate-resistant acid phosphatase (TRAP) co-localizes with receptor activator of NF-KB ligand (RANKL) and osteoprotegerin (OPG) in lysosomal-associated membrane protein 1 (LAMP1)-positive vesicles in rat osteoblasts and osteocytes. Histochem Cell Biol. 2015;143(2):195-207.

41. Zhou M, Ma J, Chen S, Chen X, Yu X. MicroRNA-17-92 cluster regulates osteoblast proliferation and differentiation. Endocrine. 2014;45(2):302-10.

42. Kang JM, Han M, Park IS, et al. Adhesion and differentiation of adiposederived stem cells on a substrate with immobilized fibroblast growth factor. Acta Biomater. 2012;8(5):1759-67.

43. Sun G, Peng H. HIF-1alpha-induced microRNA-210 reduces hypoxia-induced osteoblast MG-63 cell apoptosis. Biosci Biotechnol Biochem. 2015;79(8):1232-9.

44. Cui M, Kanemoto S, Cui X, Kaneko M, Asada R, Matsuhisa K, Tanimoto K, Yoshimoto Y, Shukunami C, Imaizumi K. OASIS modulates hypoxia pathway activity to regulate bone angiogenesis. Sci Rep. 2015;5:16455.

45. Kim BJ, Lee YS, Lee SY, et al. Osteoclast-secreted SLIT3 coordinates bone resorption and formation. J Clin Invest. 2018;128(4):1429-41.

46. Bai F, Chen X, Yang H, Xu HG. Acetyl-11-keto-beta-Boswellic acid promotes osteoblast differentiation by inhibiting tumor necrosis factor-alpha and nuclear factor-kappaB activity. J Craniofac Surg. 2018; Epub ahead of print.

47. Xi JC, Zang HY, Guo LX, et al. The PI3KJAKT cell signaling pathway is involved in regulation of osteoporosis. J Recept Signal Transduct Res. 2015; 35(6):640-5

48. Lu XL, Huo B, Chiang V, Guo XE. Osteocytic network is more responsive in calcium signaling than osteoblastic network under fluid flow. J Bone Miner Res. 2012;27(3):563-74.

49. Harimoto K, Yoshida Y, Yoshihara K, Nagaoka N, Matsumoto T, Tagawa Y. Osteoblast compatibility of materials depends on serum protein absorbability in osteogenesis. Dent Mater J. 2012;31(4):674-80.

50. Zenata $O$, Vrzal R. Fine tuning of vitamin $D$ receptor ( $V d r)$ activity by posttranscriptional and post-translational modifications. Oncotarget. 2017;8(21): 35390-402.

51. Li R, Li X, Zhou M, et al. Quantitative determination of matrix Gla protein (MGP) and BMP-2 during the osteogenicdifferentiation of human periodontal ligament cells. Arch Oral Biol. 2012;57(10):1408-17. 
52. Wang $\mathrm{S}$, Zhou $\mathrm{Y}$, Andreyev $\mathrm{O}$, et al. Overexpression of FABP3 inhibits human bone marrow derived mesenchymal stem cellproliferation but enhances their survival in hypoxia. Exp Cell Res. 2014;323(1):56-65.

53. Gajos-Michniewicz A, Pawlowska E, Ochedalski T, Piastowska-Ciesielska A. The influence of follistatin on mechanical properties of bone tissue in growing mice with overexpression of follistatin. J Bone Miner Metab. 2012;30(4):426-33.

54. Zocchi E, Daga A, Usai C, et al. Expression of CD38 increases intracellular calcium concentration and reduces doubling time in HeLa and 3 T3 cells. J Biol Chem. 1998;273(14):8017-24.

55. Kaneto CM, Lima PS, Zanette DL, et al. COL1A1 and miR-29b show lower expression levels during osteoblast differentiation of bone marrow stromal cells from osteogenesis imperfecta patients. BMC Med Genet. 2014;15:45.

56. Gao X, Ge J, Li W, Zhou W, LncRNA XL. KCNQ1OT1 promotes osteogenic differentiation to relieve osteolysis via Wnt/beta-catenin activation. Cell Biosci. 2018:8:19

57. Yang X, Zhang Y, Li Y, Wen T. MALAT1 enhanced the proliferation of human osteoblasts treated with ultrahigh molecular weight polyethylene by targeting VEGF via miR225p. Int J Mol Med. 2018;41(3):1536-46.

58. Misawa A, Orimo H. IncRNA HOTAIR inhibits mineralization in osteoblastic osteosarcoma cells by epigenetically repressing ALPL. Calcif Tissue Int 2018. Epub ahead of print.

59. Tang Z, Gong Z, Sun X. LncRNA DANCR involved osteolysis after total hip arthroplasty by regulating FOXO1 expression to inhibit osteoblast differentiation. J Biomed Sci. 2018;25(1):4.

60. Bu Y, Zheng D, Wang L, Liu J. LncRNA TSIX promotes osteoblast apoptosis in particle-induced osteolysis by down-regulating miR-30a-5p. Connect Tissue Res. 2017:1-8.

61. De-Ugarte L, Yoskovitz G, Balcells S, et al. MiRNA profiling of whole trabecular bone: identification of osteoporosis-related changes in MiRNAs in human hip bones. BMC Med Genet. 2015;8:75.

62. Wu Q, Zhou W, Feng Q, Liu X, Xiong Y, Li H. MicroRNA-92b promotes cell proliferation and invasion in osteosarcoma by directly targeting Dickkopfrelated protein 3. Exper Therap Med. 2018;15(1):173-81.

63. Gu Y, Ma L, Song L, Li X, Chen D, Bai X. miR-155 inhibits mouse osteoblast differentiation by suppressing SMAD5 expression. Biomed Res Int. 2017; 2017:1893520.

64. Myers EW, Miller W. Approximate matching of regular expressions. Bull Math Biol. 1989;51(1):5-37.

Ready to submit your research? Choose BMC and benefit from:

- fast, convenient online submission

- thorough peer review by experienced researchers in your field

- rapid publication on acceptance

- support for research data, including large and complex data types

- gold Open Access which fosters wider collaboration and increased citations

- maximum visibility for your research: over $100 \mathrm{M}$ website views per year

At BMC, research is always in progress.

Learn more biomedcentral.com/submissions 